\title{
The Gamma-ray Large Area Space Telescope (GLAST)
}

\author{
Taro Kotani ${ }^{1}$ \\ NASA/Goddard Space Flight Center, Code 661, Greenbelt, MD 20771, \\ U.S.A. \\ Present address: Tokyo Institute of Technology, Meguro-ku Oookayama \\ 2-12-1, Tokyo 152-8551, Japan
}

\begin{abstract}
GLAST, the next U.S. general gamma-ray astrophysics mission scheduled to be launched into low Earth orbit in April, 2006, for 5-10 years of operation, is described. A product of a NASA/DOE and international collaboration, the Large Area Telescope (LAT) is the primary instrument that covers the $<20 \mathrm{MeV}$ to $>300 \mathrm{GeV}$ band with an effective area $>8000 \mathrm{~cm}^{2}$. The angular resolution ranges from $<3.5^{\circ}$ at $100 \mathrm{MeV}$ to $<0.15^{\circ}$ at $10 \mathrm{GeV}$. The GLAST Burst Monitor (GBM) consists of a group of $\mathrm{NaI}$ and BGO detectors to extend GLAST's sensitivity to gamma-ray bursts to the $<10 \mathrm{keV}$ to $>25 \mathrm{MeV}$ band. GLAST's localizations enables us to identify the $\mathrm{X}$-ray, optical and radio counterparts of thousands of gamma-ray sources and to determine their nature.
\end{abstract}

\section{Overview}

GLAST is the next US general gamma-ray astrophysics mission, which is scheduled to be launched into low Earth orbit in March, 2006, for 5-10 years of operation.

A product of a NASA/DOE/international collaboration, the Large Area Telescope (LAT) will be the primary instrument that will cover the $<20 \mathrm{MeV}$ to $>300 \mathrm{GeV}$ band with an effective area $>8000 \mathrm{~cm}^{2}$. The angular resolution will range from $<3.5^{\circ}$ at $100 \mathrm{MeV}$ to $<0.15^{\circ}$ at $10 \mathrm{GeV}$. The field of view will be $>2$ sr.

The GLAST Burst Monitor (GBM) will consist of a group of NaI and BGO detectors to extend GLAST's sensitivity to gamma-ray bursts to the $<10 \mathrm{keV}$ to $>25 \mathrm{MeV}$ band.

\section{The LAT}

The upper part of the LAT will be a series of tungsten foils above silicon strip detectors (SSDs). A gamma ray entering from above will hit one of the foils, and pair-produce. A simulation event is shown in Fig. 1. The resulting $\mathrm{e}^{+} \mathrm{e}^{-}$

\footnotetext{
${ }^{1}$ For the entire GLAST mission team
} 


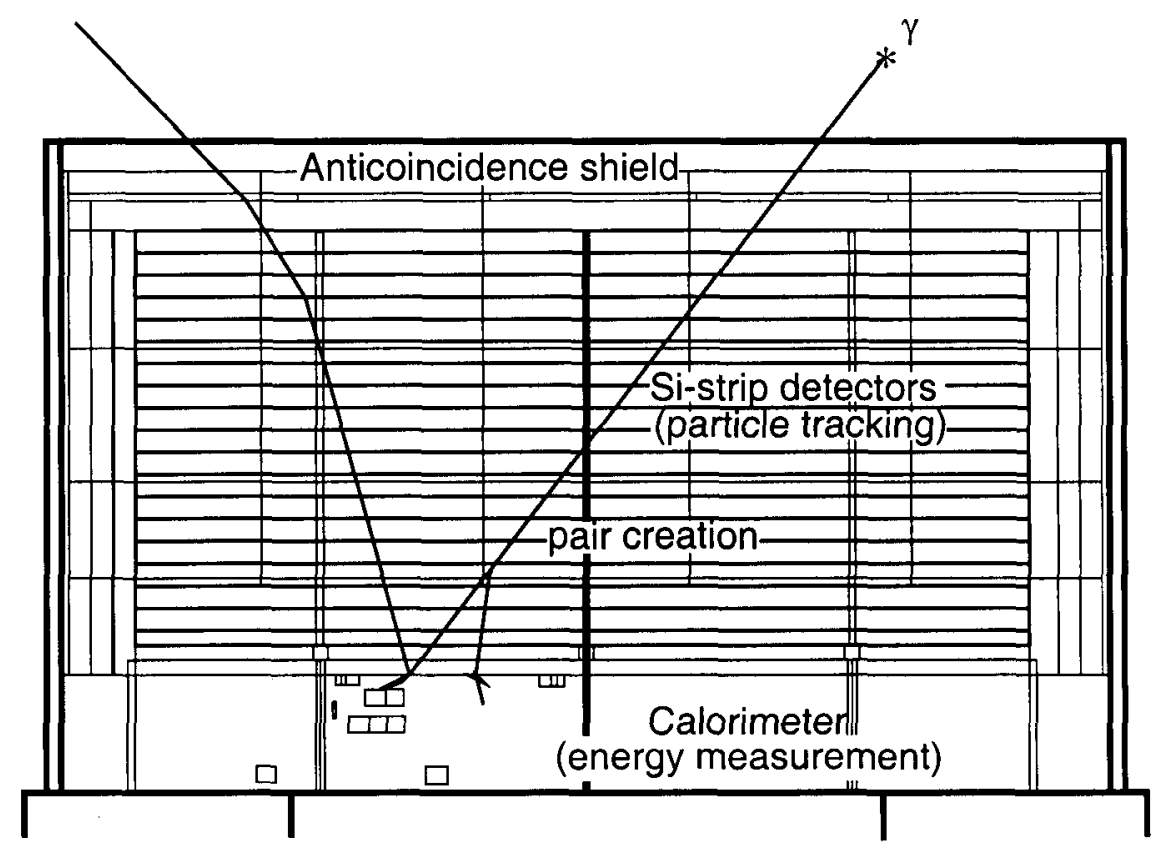

Figure 1. A simulated gamma-ray event detected with the LAT

pair will be tracked by the SSDs, providing the incident direction of the gamma ray. The $\mathrm{e}^{+} \mathrm{e}^{-}$pair will continue into the calorimeter ("logs" of CsI(Tl)); the sum of the energy deposited in the calorimeter logs will give the gamma rays' energy. Anticoincidence shields will reject charged particles.

The LAT will consist of 16 "towers." In each tower there will be 18 planes with SSDs. The SSDs will actually be two planes of strips running in crossing directions. The upper 12 planes will have thin (0.035 radiation lengths) tungsten foils, the next 4 will have thick ( 0.18 radiation lengths) tungsten foils, and the last 2 will not have any foils. Below the SSD planes will be 8 planes with 12 $\mathrm{CsI}(\mathrm{Tl})$ each. The anticoincidence shields will be plastic scintillator tiles on the outside of the 16 towers. The outside dimension of the LAT will be $1.8 \times 1.8 \times 1 \mathrm{~m}$, and it will weigh $\sim 3000 \mathrm{~kg}$.

The celestial gamma rays will be only a small fraction of the total number of events in the LAT, most of which will result from charged particles. Therefore, filtering of the events on board will reduce the $\sim 30 \mathrm{kHz}$ event rate to $\sim 30 \mathrm{~Hz}$ that will be telemetered to the ground; ground processing will result in a $\sim 1.5 \mathrm{~Hz}$ photon rate.

\section{The GBM}

A descendant of the BATSE detectors, the GBM will consist of $12 \mathrm{NaI}(\mathrm{Tl})$ detectors for the $10-1000 \mathrm{keV}$ band and 2 BGO detectors for the $1-20 \mathrm{MeV}$ band. The GBM will monitor $>8 \mathrm{sr}$ of the sky, including the LATs field-of-view. 
Bursts will be localized to $9^{\circ}$ ( $1 \sigma$, brightest $40 \%$ of the bursts) by comparing the rates in different detectors. Occultation monitoring of the sky will be feasible but is not currently funded.

\section{Operations}

After a 60 day checkout phase, GLAST will undertake a 1 year sky survey while the LAT team calibrates the instrument. The observing plan for subsequent years will be driven by guest investigations. While pointed observations will be feasible, continued survey mode operation will usually be most efficient, and will predominate. All operational modes may be interrupted for targets of opportunity. GLAST will autonomously point at the location of strong gamma-ray bursts it detects for 5 hours.

\section{Sensitivity}

The LAT will have a $5 \sigma$ sensitivity for $>100 \mathrm{MeV}$ of $<6 \times 10^{-9} \mathrm{ph} \mathrm{cm}^{-2} \mathrm{~s}^{-1}$ after 1 year. The timing accuracy will be $<10 \mu \mathrm{s}$, with a deadtime per event of $<100 \mu$ s.

Of the 271 sources in the 3rd EGRET Catalog, 172 were unidentified. With the LAT's greater sensitivity ( $>30 \times$ EGRET) and smaller PSF, the identification of gamma-ray sources will be much more secure with better source localizations by GLAST.

\section{Expected Science}

\subsection{AGN}

EGRET discovered highly variable emission from blazars, thought to be AGNs with their jets pointed at us. The gamma rays are probably low energy photons inverse Compton scattered by the jets. EGRET detected $\sim 85$ AGN, GLAST should detect $\sim 4500$. GLAST will monitor sub-day variability for bright sources, resolve daily variability for medium sources, determine the blazar luminosity function, and localize sources. GLAST's observations will overlap the higher energy ground-based observations.

\subsection{Gamma-Ray Bursts}

EGRET detected $\mathrm{E}>100 \mathrm{MeV}$ photons from 6 bursts. In 2-3 cases this emission lingered beyond the end of the lower energy lightcurve. The EGRET observations were severely deadtime limited. Both the GBM and the LAT will detect and localize bursts, alert the ground within $7 \mathrm{~s}$, and repoint GLAST toward strong bursts for $\sim 5$ hours. Together the GBM and the LAT will observe the spectrum from $10 \mathrm{keV}$ to $300 \mathrm{GeV}$ ! Both afterglows and emission during the burst are expected. 


\subsection{Extragalactic Absorption}

Gamma rays from AGN and GRBs pair-produce on photons from the diffuse infrared background. The strength of this background can be measured from the resulting breaks in the source spectrum.

\subsection{Diffuse Emission}

The extragalactic emission most likely is the emission of faint AGN; GLAST should resolve a large fraction of this background. The Galactic background results from cosmic ray-gas interactions. These backgrounds must be modeled for analysis of superimposed point sources.

\subsection{Dark Matter Particles}

The particles that make up the dark matter may annihilate or decay, resulting in narrow lines, possibly observable by GLAST.

\subsection{Pulsars}

EGRET detected 7 pulsars, with 5 candidates. As opposed to the radio emission, the gamma rays are a major fraction of the pulsar's energy loss. The outstanding question is whether the emission originates from the polar cap or the "outer gap" where open field lines pass through the light cylinder.

\section{Gamma Rays from Binary Systems}

The 170 unidentified sources in the 3rd EGRET Catalog remain an astrophysical mystery. About 30 of them reside at high galactic latitude $\left(>30^{\circ}\right)$ and are probably AGNs. The rest are located in the galactic plane and are assumed to be galactic objects. Proposed candidates include pulsars, supernova remnants, molecular clouds, and binary systems. Although few binary systems have been positively identified as gamma-ray sources so far, some binary systems may be responsible for gamma-ray emission, as shown below. GLAST's excellent localizations will identify the X-ray, optical and radio counterparts of both the unidentified EGRET sources and the thousands of new sources GLAST will detect, and will determine their nature. If binary systems are confirmed as gamma-ray sources, a new class of astrophysical object would be established.

\subsection{Microquasars}

Microquasars such as SS 433, GRS 1915+105, and GRO J1655-40, can emit gamma rays via inverse Comptonization by the high-energy electrons in their relativistic jet. As is thought to occur in AGN, the gamma-ray emission may be Doppler-boosted ( $\gamma \sim \mathrm{a}$ few) in the jet's direction resulting in detectable emission only for observers looking down the jet (Kaufman Bernadó, Romero, $\&$ Mirabel 2002). Thus GLAST may detect microquasars with their jet aligned with the line of site. 


\subsection{Ejecta from Microquasars}

SS 433's X-ray jet lobes are synchrotron sources (Yamauchi, Kawai, \& Aoki 1994; Safi-Harb \& Petre 1999) suggesting the presence of high-energy electrons accelerated by the relativistic shock. The electrons can emit gamma rays by inverse Comptonization of the cosmic microwave background radiation or piondecay process. For example, the flux from SS 433's jet lobe is estimated to be $\sim 10-10 \mathrm{ph} \mathrm{cm}^{-2} \mathrm{~s}^{-1}$ (30 MeV-300 GeV), which is, unfortunately, below the detection limit of GLAST. GLAST might find jet sources similar to SS 433 or the historical activity of jet sources from their ejecta. The detection of supernova remnants is also possible.

\subsection{Colliding Stellar Winds}

Super-critical accretion accompanies a mass loss in the form of disk wind (Paragi et al. 1999). The collision of the disk wind and the stellar wind from the companion star can shock-accelerate electrons and produce gamma rays by inverse Comptonization, synchrotron, and pion-decay processes.

\section{References}

Hartman, R. C., et al. 1999, ApJS, 123, 79

Kaufman Bernadó, M. M., Romero, G. E., \& Mirabel, I. F. 2002, A\&A, 385, L10

Paragi, Z., Vermeulen, R. C., Fejes, I., Schilizzi, R. T., Spencer, R. E., \& Stirling, A. M. 1999, A\&A, 348, 910

Safi-Harb, S., \& Petre, R. 1999, ApJ, 512, 784

Yamauchi, S., Kawai, N., \& Aoki, T. 1994, PASJ, 46, L109 